23 Ruusuvaara L. Teenage abortions, family backgrotend, scxual experience and contraceptive use. [dissertation]. Helsinki: Lniversity of Helsinki, 1983.

24 Holmgren K. Stigande aborttal i Sverige. Dags för sexologispecialitet? Nord Med 1990;105:46-8

25 Sosiaali- ja tervevshallitus. Tilastojulkaisu 1991. Tartuntatautitilann ruosina 1988-1990 ja rokotukset zuosina 1987-1990 Suomessa. Helsinki: Oy Ylipistopaino, 1991.

26 DeBuono B, Zinner S, Daamen M, McCormack W. Sexual behavior of college women in 1975, 1986, and 1989. N Engl f.Med 1990:322:821-5.

7 Thorogod $\mathrm{M}$ Mann J Murphy $\mathrm{I}$ Vessey $\mathrm{M}$ Risk factors for fatal venous hroge

thromboembism in

feriew. $\mathrm{Br}$ Obstet Grnaecol 1987;94:724-30

Beral V, Hannaford P. Clifford K. Oral contraceptive use and malignancies of the genital tract. Lancet 1988 ;ii:1331-5.

30 Brinton LA. Oral contraceptives and cervical ncoplasia. Contraception 1991; 43:581-95.
31 Hirvonen E, Idänpää-Heikkilä J. Cardiovascular death among women under 40 years of age using low-estrogen oral contraceptives and intrauterine devices in Finland from 1975 to 1984. Am f Obstet Gynecol 1990;163:281-4.

32 Wallace H, Vienonen M. Teenage pregnancy in Sweden and Finland Implications for the United States. F Adolesc Health Care 1989:10:231-6.

33 Pike MC, Henderson BE, Krailo MD, Duke A, Roy S. Breast cancer in young women and use of oral contraceptives: possible modifying effects of formulation and age at use Lancet 1983;ii:926-30.

34 Mandelin M. Stabilt antal aborter i Finland. Nord Med 1990;105:41

35 Tikkanen J, Koskela K, Haikala O. Finnish response to human immunodeficiency virus infection. Hugic 1988:77:28-31.

36 Jones EF, Forrest JD, Goldman N, Hensaw SK, Lincoln R, Rosoff JI, et al. Teenage pregnancy in developed countries: determinants and policy implications. Fam Plann Perspect 1985;17:53-63.

Accopted 3 Septement 1992)
Department of Medicine,

Muhimbili Medical Centre,

University of Dar es

Salaam, Dar es Salaam,

Tanzania

Andrew B Swai, senior

lecturer in medicine

Donald G McLarty, professor

of medicine

Ministry of Health, Dar es

Salaam, Tanzania

Henry M Kitange, specialist physician

Gabriel Masuki, district

medical officer

Peter M Kilima, community physician

Human Diabetes and Metabolism Research Centre, University of Newcastle upon Tyne, Newcastle upon Tyne NE2 4HH

K George M M Alberti, professor of medicine

Correspondence to: Professor Alberti.

BM7 1992;305:1057-62

\title{
Is diabetes mellitus related to undernutrition in rural Tanzania?//
}

\author{
Andrew B_Swai, Henry M_Kitange, Gabriel Masuki, Peter MLKilima, K George M M,Alberti, \\ Donald GMcLarty
}

Abstract

Objective-To investigate the relation between undernutrition and diabetes.

Design-Survey of glucose tolerance in rural Tanzania.

Setting-Eight villages in three widely separated regions of Tanzania.

Subjects-8581 people aged 15 and above: 3705 men and 4876 women.

Main outcome measures-Oral glucose tolerance, body mass index, height, and low haemoglobin and cholesterol concentrations.

Results-In the eight villages $42 \cdot 7-56 \cdot 9 \%$ of all men and $30 \cdot 0-45 \cdot 2 \%$ of all women had a body mass index below $20 \mathrm{~kg} / \mathrm{m}^{2}$; the lowest quintile was $18.2 \mathrm{~kg} / \mathrm{m}^{2}$ in men and $18.6 \mathrm{~kg} / \mathrm{m}^{2}$ in women. The prevalence of diabetes did not change significantly from the lowest to the highest fifths of body mass index in men (lowest $1.6 \%$ (95\% confidence interval $0.8 \%$ to $2 \cdot 9 \%$ ) $v$ highest $1 \cdot 3 \%(0.7 \%$ to $2 \cdot 5 \%)$ ) or women $(1 \cdot 1 \%(0 \cdot 6 \%$ to $2 \cdot 1 \%) v 0 \cdot 5 \%(0 \cdot 2 \%$ to $1 \cdot 2 \%))$. In men and in women prevalence of impaired glucose tolerance was greater in the lowest fifths of height $(8 \cdot 2 \%(6 \cdot 3 \%$ to $10 \cdot 6 \%)$, and $11 \cdot 1 \%(9 \cdot 2 \%$ to $13 \cdot 3 \%)$ ) respectively and body mass index $(9 \cdot 6 \%$ $(7 \cdot 5 \%$ to $12 \cdot 1 \%)$, and $8 \cdot 4 \%(6 \cdot 7 \%$ to $10 \cdot 5 \%)$ than in the highest fifths (impaired glucose tolerance $\mathbf{4} \cdot \mathbf{7 \%}$ $(3 \cdot 4 \%$ to $6 \cdot 5 \%)$; and $5 \cdot 1 \%(3.9 \%$ to $6 \cdot 7 \%)$; body mass index $5 \cdot 1 \%(3 \cdot 7 \%$ to $7 \cdot 0 \%)$, and $7 \cdot 7 \%(6 \cdot 2 \%$ to $9 \cdot 6 \%)$.

Conclusion-Rates of diabetes were not significantly associated with low body mass index or height, but overall rates were much lower than those in well nourished Western populations. Increased impaired glucose tolerance in the most malnourished people may reflect the larger glucose load per kilogram weight. The role of undernutrition in the aetiology of diabetes must be questioned.

\section{Introduction}

In 1985 the World Health Organisation expert committee on diabetes mellitus introduced malnutrition related diabetes mellitus as "a major clinical subclass, ranking with insulin dependent diabetes mellitus and non-insulin dependent diabetes mellitus." Bajaj suggested that there are at least two subclasses of malnutrition related diabetes mellitus: fibrocalculous pancreatic diabetes and protein deficient pancreatic diabetes, and this classification has been adopted by the WHO.' Several reviews of malnutrition related diabetes have been published. ${ }^{3-7}$ Most have favoured the view that chronic undernutrition is a key factor in the causation of malnutrition related diabetes mellitus, but direct evidence is poor and we have questioned the existence of the protein deficient form of malnutrition related diabetes mellitus.

Traditionally a low body weight and energy intake were considered to decrease the risk of diabetes. ${ }^{\circ}$ This view was based on observations such as the reduced incidence of diabetes mellitus in Europe during the first and second world wars ${ }^{4}$ and the observation that the prevalence of non-insulin dependent diabetes in different countries was positively related to the "average fatness" of the population." The same association has been observed in intrapopulation studies. Gupta et al found that, among urban Indians, rates of diabetes were $3.6 \%$ in subjects with a normal body weight and $1.5 \%$ in very lean subjects." On the other hand, Rao cited the results of a large study in India in which rates of diabetes were not significantly different between urban and rural subjects as evidence pointing to an association between diabetes and undernutrition, since most rural subjects were undernourished. ${ }^{+}$In general, diabetes is less common in undernourished populations than in well nourished populations. ${ }^{12}$ This does not, however, answer the question clearly whether within the same population chronic undernutrition leads to a clinically and statistically significant increase in diabetes. We attempted to answer this question by studying glucose tolerance in rural African communities with a background of malnutrition.

\section{Patients and methods}

The study was carried out in eight villages in Tanzania and forms part of a long term programme aimed at reducing morbidity and mortality with continuing care. (Treatment was offered to all those found to be ill during surveys.) Six villages were chosen at random in two contrasting regions: Kilimanjaro, one of the most prosperous, and Morogoro region, one of the more economically disadvantaged..$^{13}$ Of the remaining two villages, one in Mara region was chosen because the inhabitants had been exposed to high concentrations of dietary cyanide, and the other in Kilimanjaro region was chosen because hypertension was thought to be common. In four of the eight villages the total population aged 15 years and over was about 1000 . The entire adult population in these villages was therefore invited to participate in the study. In the other villages a list of the names of the leaders of the 10 cell units (10 families) was obtained and the number of units required to provide about 1000 subjects was selected at random. Slightly more people were selected in Uswaa village. Lengthy discussions were held with village leaders before the start of the study to ensure 
high rates of compliance. Table I shows that village sample sizes ranged from 899 (Mdawi) to 1430 (Uswaa) with response rates of $72.5 \%$ to $96 \%$. Also shown are age and gender and the main staple carbohydrates consumed. Both Morogoro and Kilimanjaro regions are regions of agricultural importance, but the per capita income and meat consumption of peasant farmers in Kilimanjaro is higher than that of peasants in Morogoro. Childhood malnutrition is common in both regions despite these differences. Since information on the nutritional state of children is important when investigating a possible relation between glucose intolerance and undernutrition we attempted to document information on the nutritional state of children in the villages and districts where the surveys were carried out.

\section{NUTRITION}

The overall prevalence of protein energy malnutrition in rural Tanzania varies between $30 \%$ and $80 \%$, with half of the under 5 year old population being undernourished in most areas. " If undernutrition is assessed by height for age, about $60 \%$ of children are stunted during their fourth year, and even among older children rates of stunting remain around $30 \%$. Studies have shown that most people receive only about $60 \%$ of their daily protein requirements. ${ }^{15}$

The nutrition and health of children in one village in Morogoro region with similar characteristics to Msolwa (one of the villages in Morogoro in this study) have been studied extensively." A high degree of stunting $(35-71 \%)$ was found among children aged 1 month to 15 years and wasting in $3-20 \%$. The infant mortality rate was 198 per 1000 births, which was much higher than the national average of 137 per 1000 .

TABL.1: I-Age and sex distribution of subjects studicd in cight villages

\begin{tabular}{lccccl}
\hline Village & $\begin{array}{c}\text { No of } \\
\text { subjects invited }\end{array}$ & $\begin{array}{c}\text { No("\%) } \\
\text { responding }\end{array}$ & $\begin{array}{c}\text { No (\%) } \\
\text { men }\end{array}$ & $\begin{array}{c}\text { Mean age } \\
\text { (vears) }\end{array}$ & Staple carbohydrate \\
\hline Madazini & 1346 & $1141(84 \cdot 8)$ & $567(49 \cdot 7)$ & 34 & Maize, rice \\
Mellela & 1337 & $969(72 \cdot 5)$ & $414(42 \cdot 7)$ & 38 & Millet \\
Msolwa & 1027 & $976(95 \cdot 0)$ & $492(50 \cdot 4)$ & 34 & Maize, rice, cassava \\
Mdawi & 930 & $899(96 \cdot 0)$ & $309(34 \cdot 4)$ & 41 & Banana \\
Naibili & 1155 & $1090(94 \cdot 4)$ & $462(42 \cdot 4)$ & 36 & Banana, maize, rice \\
Usari & 1109 & $1008(90 \cdot 9)$ & $379(37 \cdot 6)$ & 38 & Banana \\
Uswaa & 1505 & $1430(95 \cdot 0)$ & $601(42 \cdot 0)$ & 41 & Banana \\
Nyambori & 1424 & $1068(75 \cdot 0)$ & $482(45 \cdot 1)$ & 34 & Cassava \\
\hline
\end{tabular}

A nutritional survey sponsored by UNICEF was carried out among children in Usari village, one of our study villages, in Kilimanjaro region during September-October 1987. Thirty seven per cent of the children were found to be malnourished."

METHODS

Subjects participating in the surveys were requested to report to the village school or dispensary after an overnight fast. Venous blood samples were taken fasting and, except for known diabetic patients, two hours after a $75 \mathrm{~g}$ oral glucose load. A beam balance was used to measure weight without shoes or coats and height was measured in bare feet. Age and sex were recorded on a questionnaire.

Portions of the fasting and two hour blood glucose samples were drawn into fluoride tubes and analysed at the survey site with a glucose analyser (Yellow Springs Instruments, Ohio, United States) with appropriate quality control measurements as previously described. ${ }^{13}$ An aliquot of sample was also kept frozen at $-20^{\circ} \mathrm{C}$ for analysis of serum cholesterol by an enzymatic method ${ }^{1 *}$ with the Cobas Bio centrifugal analyser (Roche, Welwyn Garden City). Serum cholesterol concentration was used to provide a crude indication of fat and animal protein intake. Haemoglobin was also used as an indicator of nutritional status and was measured with a BMS haemoglobinometer (Buffalo Medical Specialties, Buffalo, United States).

\section{DEFINITIONS}

Diabetes was defined according to the 1985 WHO criteria'-that is, two hour blood glucose concentration $\geqslant 10.0 \mathrm{mmol} / 1$ for diabetes and $\geqslant 6.7 \mathrm{mmol} / 1$ but $<10 \mathrm{mmol} / 1$ for impaired glucose tolerance. For diagnosis of diabetes at least two abnormal blood glucose values were required in an asymptomatic person but for epidemiological studies a single diagnostic value was acceptable. All the subjects with diabetes already diagnosed at the time of study had fasting blood glucose concentrations $\geqslant 6.7 \mathrm{mmol} / \mathrm{l}$ and were defined as diabetic irrespective of their current treatment. Body mass index was defined as weight $(\mathrm{kg})$ divided by the square of height $(\mathrm{m})$. Patients with a body mass index below $20 \mathrm{~kg} / \mathrm{m}^{2}$ were regarded as underweight, those with an index $20-24.9 \mathrm{~kg} / \mathrm{m}^{2}$ as

ТАВ1.1: II-Distribution of haemoglobin and cholesterol concentrations and body mass index in men in eight villages. Values are numbers (percentages) unless stated otherwise

\begin{tabular}{|c|c|c|c|c|c|c|c|c|c|c|}
\hline \multirow[b]{2}{*}{ Village } & \multicolumn{4}{|c|}{ Hacmoglobin $(\mathrm{g} / \mathrm{l})$} & \multicolumn{3}{|c|}{ Cholesterol $(\mathrm{mmol} / \mathrm{l})$} & \multicolumn{3}{|c|}{ Body mass index $(\mathrm{kg} / \mathrm{m})$} \\
\hline & $<110$ & $110-129$ & 130 & Mean (SD) & $<3 \cdot 3^{\star}$ & $>5 \cdot 2$ & Mean (SD) & $20 \cdot 0$ & $>25 \cdot 0$ & Mean (SD) \\
\hline Madazini & $157(27 \cdot 8)$ & $255(45 \cdot 0)$ & $155(27 \cdot 3)$ & $118(18)$ & $258(45.5)$ & $35(6 \cdot 1)$ & $3 \cdot 5(1 \cdot 0)$ & $242(42 \cdot 7)$ & $19(3 \cdot 4)$ & $20 \cdot 4(2 \cdot 4)$ \\
\hline Melela & $92(22 \cdot 1)$ & $208(50 \cdot 2)$ & $114(27 \cdot 6)$ & $118(17)$ & $263(63.6)$ & $7(1 \cdot 7)$ & $3.0(0.8)$ & $191(46 \cdot 1)$ & $7(1 \cdot 7)$ & $20 \cdot 1(2 \cdot 2)$ \\
\hline Msolwa & $165(33.5)$ & $227(46 \cdot 2)$ & $100(20 \cdot 3)$ & $115(18)$ & $202(41 \cdot 0)$ & $29(5.9)$ & $3.6(1.0)$ & $248(50 \cdot 4)$ & $8(1 \cdot 6)$ & $19 \cdot 9(2 \cdot 2)$ \\
\hline Mdawi & $12(4 \cdot 0)$ & $107(34 \cdot 7)$ & $190(61 \cdot 3)$ & $132(15)$ & $19(6 \cdot 2)$ & $117(38 \cdot 0)$ & $5 \cdot 0(1 \cdot 3)$ & $176(56.9)$ & $13(4 \cdot 3)$ & $19 \cdot 6(2 \cdot 8)$ \\
\hline Naibili & $11(2 \cdot 4)$ & $73(15.8)$ & $378(81 \cdot 8)$ & $142(17)$ & $75(16 \cdot 3)$ & $122(26 \cdot 3)$ & $4 \cdot 5(1.3)$ & $209(45 \cdot 3)$ & $17(3 \cdot 6)$ & $20 \cdot 3(2 \cdot 4)$ \\
\hline Usari & $7(1.9)$ & $25(6 \cdot 7)$ & $347(91 \cdot 5)$ & $151(19)$ & $115(30 \cdot 3)$ & $32(8 \cdot 4)$ & $3.9(1 \cdot 1)$ & $180(47 \cdot 6)$ & $13(3 \cdot 5)$ & $20 \cdot 2(2 \cdot 3)$ \\
\hline Uswaa & $32(5.3)$ & $290(48 \cdot 2)$ & $279(46 \cdot 4)$ & $128(14)$ & $156(25.9)$ & $62(10 \cdot 3)$ & $4 \cdot 0(1 \cdot 0)$ & $279(46 \cdot 4)$ & $28(4 \cdot 7)$ & $20 \cdot 3(2 \cdot 4)$ \\
\hline Nyambori & $131(27 \cdot 3)$ & $261(54 \cdot 2)$ & $90(18 \cdot 6)$ & $116(14)$ & $145(30 \cdot 0)$ & $28(5 \cdot 8)$ & $3.7(0.9)$ & $241(49 \cdot 8)$ & $14(2.9)$ & $20 \cdot 1(2 \cdot 5)$ \\
\hline Total & & & & $126(21)$ & & & $3.9(1.2)$ & & & $20 \cdot 1(2 \cdot 4)$ \\
\hline
\end{tabular}

*Two standard deviations below the mean for British subjects.

TAB1.: III-Distribution of haemoglobin and cholesterol concentrations and body mass index in women in cight villages. Values are numbers (percentages) unless stated otherwise

\begin{tabular}{|c|c|c|c|c|c|c|c|c|c|c|}
\hline \multirow[b]{2}{*}{ Village } & \multicolumn{4}{|c|}{ Hacmoglobin $(\mathrm{g} / \mathrm{l})$} & \multicolumn{3}{|c|}{ Cholesterol $(\mathrm{mmol} / \mathrm{l})$} & \multicolumn{3}{|c|}{ Body mass index $\left(\mathrm{kg} / \mathrm{m}^{\prime}\right)$} \\
\hline & $<110$ & $100-119$ & 120 & Mean (SD) & $3 \cdot 3^{\star}$ & $>5 \cdot 2$ & Mean $(\mathrm{SD})$ & $<20 \cdot 0$ & $>25 \cdot 0$ & Mean (SI) \\
\hline Madazini & $199(34 \cdot 6)$ & $318(55 \cdot 5)$ & $57(9 \cdot 9)$ & $101(15)$ & $200(34 \cdot 9)$ & $40(7 \cdot 0)$ & $3 \cdot 7(1 \cdot 0)$ & $172(30 \cdot 0)$ & $38(6 \cdot 6)$ & $21 \cdot 6(3 \cdot 2)$ \\
\hline Melela & $122(22 \cdot 0)$ & $296(53.4)$ & $137(24 \cdot 6)$ & $107(15)$ & $330(59 \cdot 4)$ & $7(1 \cdot 2)$ & $3 \cdot 2(0 \cdot 8)$ & $251(45 \cdot 2)$ & $37(6 \cdot 6)$ & $20 \cdot 6(2 \cdot 8)$ \\
\hline Msolwa & $112(23 \cdot 1)$ & $246(50 \cdot 8)$ & $126(26 \cdot 0)$ & $108(15)$ & $151(31 \cdot 3)$ & $53(10 \cdot 9)$ & $3.9(1 \cdot 1)$ & $200(41 \cdot 5)$ & $24(5 \cdot 0)$ & $20 \cdot 6(2 \cdot 6)$ \\
\hline Mdawi & $20(3 \cdot 3)$ & $177(30 \cdot 1)$ & $393(66 \cdot 6)$ & $122(14)$ & $36(6 \cdot 1)$ & $237(40 \cdot 2)$ & $5 \cdot 0(1 \cdot 3)$ & $248(42 \cdot 0)$ & $45(7 \cdot 7)$ & $20 \cdot 7(3 \cdot 1)$ \\
\hline Naibili & $18(2 \cdot 8)$ & $110(17 \cdot 6)$ & $500(79 \cdot 6)$ & $130(16)$ & $90(1+4)$ & $157(25 \cdot 0)$ & $4.5(1.3)$ & $239(38 \cdot 0)$ & $67(10 \cdot 6)$ & $21 \cdot 3(3 \cdot 0)$ \\
\hline Usari & $5(0.8)$ & $+3(6.8)$ & $581(92.4)$ & $139(16)$ & $118(18.8)$ & $60(9 \cdot 6)$ & $4.0(0.9)$ & $232(36.9)$ & $61(9 \cdot 7)$ & $21 \cdot 1(2.9)$ \\
\hline Uswaa & $17(2 \cdot 0)$ & $286(34.5)$ & $526(63.5)$ & $122(12)$ & $122(14 \cdot 7)$ & $99(12 \cdot 0)$ & $4.2(0.9)$ & $288(34 \cdot 7)$ & $94(11 \cdot 3)$ & $21 \cdot 4(3 \cdot 1)$ \\
\hline Nyambori & $101(17 \cdot 2)$ & $367(62 \cdot 6)$ & $118(20 \cdot 2)$ & $108(13)$ & $152(25.9)$ & $41(7 \cdot 0)$ & $3.9(0.9)$ & $221(37 \cdot 7)$ & $43(7 \cdot 4)$ & $20 \cdot 8(2 \cdot 6)$ \\
\hline Total & & & & $118(19)$ & & & $4 \cdot 1(1 \cdot 2)$ & & & $21 \cdot()(2 \cdot 9)$ \\
\hline
\end{tabular}

*Two standard deviations below the mean for British subjects. 


\begin{tabular}{|c|c|c|c|c|c|c|c|c|}
\hline \multirow[b]{2}{*}{$\begin{array}{l}\text { Body mass } \\
\text { index } \\
\left(\mathrm{kg} / \mathrm{m}^{2}\right)\end{array}$} & \multicolumn{4}{|c|}{ Diabetes } & \multicolumn{4}{|c|}{ Impaired glucose tolerance } \\
\hline & $15-34$ years & $35-54$ years & $\geqslant 55$ years & $\begin{array}{c}\text { All ages } \\
\text { (95\%\% confidence } \\
\text { interval) }\end{array}$ & $15-34$ years & $35-54$ years & $\geq 55$ years & $\begin{array}{c}\text { All ages } \\
\text { (95\% confidence } \\
\text { interval) }\end{array}$ \\
\hline \multicolumn{9}{|l|}{ Men: } \\
\hline 18.2 & 0.8 & 0.7 & $4 \cdot 2$ & $1.6(0.8$ to 2.9$)$ & $5 \cdot 8$ & $8 \cdot 6$ & $19 \cdot 2$ & $9.6(7.5$ to $12 \cdot 1)$ \\
\hline$-19 \cdot 5$ & 0.3 & 0.5 & 3.5 & $1.1(0.5$ to 2.3$)$ & $5 \cdot 3$ & $6 \cdot 5$ & $15 \cdot 7$ & $9.1(6.3$ to 10.4$)$ \\
\hline$-20 \cdot 6$ & 0.6 & $0 \cdot 9$ & $3 \cdot 0$ & $1.2(0.5$ to 2.4$)$ & $4 \cdot 2$ & $6 \cdot 6$ & $9 \cdot 5$ & $6 \cdot 1(4 \cdot 5$ to $8 \cdot 1)$ \\
\hline$-21 \cdot 8$ & 0.5 & $1 \cdot 3$ & 0.8 & $0.8(0.3$ to 1.9$)$ & $2 \cdot 5$ & $6 \cdot 1$ & $9 \cdot 2$ & $4.8(3.4$ to 6.7$)$ \\
\hline$>21.9$ & $0 \cdot 3$ & $0 \cdot 7$ & $4 \cdot 8$ & $1.3(0.7$ to 2.5$)$ & $2 \cdot 0$ & $6 \cdot 6$ & 8.9 & $5.1(3.7$ to 7.0$)$ \\
\hline \multicolumn{9}{|c|}{$\begin{array}{l}\text { Total }(95 \% \\
\text { confidence }\end{array}$} \\
\hline \multicolumn{9}{|l|}{ Women: } \\
\hline$\therefore 18.6$ & 0.2 & $0 \cdot 8$ & $2 \cdot 7$ & $1 \cdot 1(0.6$ to $2 \cdot 1)$ & $4 \cdot 2$ & $9 \cdot 7$ & $13 \cdot 8$ & $8.4(6.7$ to 10.5$)$ \\
\hline$-20 \cdot 1$ & $0 \cdot 0$ & $1 \cdot 3$ & $0 \cdot \overline{5}$ & $0.5(0.2$ to 1.2$)$ & $5 \cdot 8$ & $6 \cdot 6$ & 11.9 & $7.2(5.7$ to 9.0$)$ \\
\hline$-21 \cdot 5$ & $0 \cdot 2$ & $0 \cdot 4$ & $2 \cdot 4$ & $0.5(0.2$ to 1.3$)$ & $5 \cdot 0$ & $9 \cdot 8$ & $16 \cdot 0$ & $7.8(6.2$ to 9.7$)$ \\
\hline$-23 \cdot 2$ & 0.7 & $0 \cdot 7$ & $3 \cdot 7$ & $1 \cdot 1(0 \cdot 6$ to $2 \cdot 1)$ & $4 \cdot 4$ & $6 \cdot 9$ & $10 \cdot 3$ & $5 \cdot 8(4 \cdot 4$ to $7 \cdot 6)$ \\
\hline $23 \cdot 2$ & $0 \cdot 0$ & 0.9 & 1.6 & $0.5(0.2$ to 1.2$)$ & $3 \cdot 8$ & $9 \cdot 6$ & $19 \cdot 4$ & $7 \cdot 7(6.2$ to $9 \cdot 6)$ \\
\hline
\end{tabular}

Total $(95 \%$

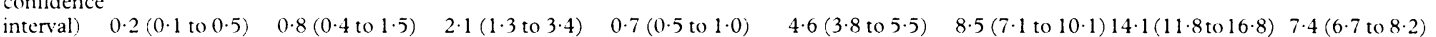

$\chi$ analysis for trends non-significant for diabetes for both genders combined $(p=0.36)$ and for genders separately; $\chi^{2}=11 \cdot 51, p<0 \cdot 0007$ for impaired glucose tolerance, genders combined.

TАВІ Е V-Crude prevalence (\%) of diabetes and impaired glucose tolerance according to height (divided by quintiles) and age groups

\begin{tabular}{|c|c|c|c|c|c|c|c|c|}
\hline \multirow[b]{2}{*}{$\begin{array}{l}\text { Height } \\
\text { (m) }\end{array}$} & \multicolumn{4}{|c|}{ Diabetes } & \multicolumn{4}{|c|}{ Impaired glucose tolerance } \\
\hline & $15-34$ years & $35-54$ years & $\geqslant 55$ years & $\begin{array}{c}\text { All ages } \\
(95 \% \text { confidence } \\
\text { interval })\end{array}$ & $15-34$ years & $35-54$ years & $\geqslant 55$ years & $\begin{array}{c}\text { All ages } \\
\text { (95\% confidence } \\
\text { interval) }\end{array}$ \\
\hline \multicolumn{9}{|l|}{ Men: } \\
\hline$<1.58$ & 0.5 & 1.5 & $4 \cdot 5$ & $1.3(0.6$ to 2.6$)$ & $5 \cdot 2$ & $11 \cdot 0$ & $16 \cdot 1$ & $8.2(6.3$ to 10.6$)$ \\
\hline-1.63 & 0.6 & $1 \cdot 3$ & $2 \cdot 9$ & $1.4(0.7$ to 2.6$)$ & $3 \cdot 7$ & $8 \cdot 6$ & $18 \cdot 9$ & $9 \cdot 3(7.4$ to 11.6$)$ \\
\hline-1.665 & 0.7 & 0.5 & $2 \cdot 4$ & $1.0(0.4$ to 2.2$)$ & $4 \cdot 3$ & $4 \cdot 5$ & 9.5 & $5.7(4 \cdot 1$ to 7.8$)$ \\
\hline$-1 \cdot 71$ & 0.6 & $0 \cdot 0$ & 3.9 & $1.1(0.5$ to 2.3$)$ & $3 \cdot 3$ & $6 \cdot 3$ & $10 \cdot 5$ & $5.8(4.2$ to 7.9$)$ \\
\hline$>1 \cdot 71$ & 0.3 & $1 \cdot 1$ & $3 \cdot 4$ & $1.1(0.5$ to 2.2$)$ & $3 \cdot 3$ & $5 \cdot 1$ & $7 \cdot 6$ & $4.7(3.4$ to 6.5$)$ \\
\hline \multicolumn{9}{|c|}{$\begin{array}{l}\text { Total }(95 \%, \\
\text { confidence }\end{array}$} \\
\hline \multicolumn{9}{|l|}{ Women: } \\
\hline 1.505 & 0.2 & $1 \cdot 1$ & $2 \cdot 7$ & $1.0(0.5$ to 1.9$)$ & $7 \cdot 1$ & $11 \cdot 4$ & $19 \cdot 2$ & $11.1(9.2$ to 13.3$)$ \\
\hline-1.545 & 0.4 & 1.0 & $3 \cdot 1$ & $1.0(0.2$ to 1.9$)$ & $5 \cdot 0$ & $7 \cdot 5$ & $14 \cdot 8$ & $7.5(6.0$ to 9.4$)$ \\
\hline-1.575 & $0 \cdot 0$ & $0 \cdot 0$ & 0.6 & $0.1(0.0$ to 0.7$)$ & $4 \cdot 6$ & $8 \cdot 7$ & $14 \cdot 6$ & $7.7(6.1$ to 9.7$)$ \\
\hline-1.615 & 0.2 & $0 \cdot 7$ & $2 \cdot 8$ & $0.7(0.3$ to 1.5$)$ & 3.5 & 8.9 & $8 \cdot 5$ & $5.7(4.3$ to 7.4$)$ \\
\hline$>1.615$ & 0.3 & $1 \cdot 3$ & 0.9 & $0.7(0.3$ to 1.5$)$ & $3 \cdot 5$ & $6 \cdot 4$ & 9.5 & $5.1(3.9$ to 6.7$)$ \\
\hline $\begin{array}{l}\text { Total }(95 \% \\
\text { confidence } \\
\text { interval) }\end{array}$ & $0.2(0 \cdot 1$ to 0.5$)$ & $0.8(0.4$ to $1 \cdot 5)$ & $2 \cdot 1(1.3$ to $3 \cdot 4)$ & $0.7(0.5$ to 1.0$)$ & $4.6(3.8$ to 5.5$)$ & $8 \cdot 5(7 \cdot 1$ to $10 \cdot 1)$ & $14 \cdot 1(11 \cdot 8$ to $16 \cdot 8)$ & $7 \cdot 4(6 \cdot 7$ to $8 \cdot 2)$ \\
\hline
\end{tabular}

normal weight, and those with an index $25 \mathrm{~kg} / \mathrm{m}^{2}$ and over as overweight. Quintiles of body mass index and height were calculated for each sex separately.

Normal values of haemoglobin concentrations for men were $130 \mathrm{~g} / \mathrm{l}$ or higher and for women $120 \mathrm{~g} / \mathrm{l}$ or higher. Since, however, the haemoglobin concentrations in both sexes in rural Africa are about $10 \mathrm{~g} / \mathrm{l}$ lower on average than the normal, ${ }^{19}$ the distribution of haemoglobin in the survey populations was divided into three categories: $<110,110-129$, and $130-170 \mathrm{~g} / 1$ for men and <100, 100-119, and 120-160 g/l for women. Cholesterol values were divided into three bands: above $5 \cdot 1 \mathrm{mmol} / \mathrm{l}$ (which is raised according to the European Atherosclerosis Society and the United States national cholesterol education programme ${ }^{2021}$ ) below $3.3 \mathrm{mmol} / \mathrm{l}$ (2 SD below the mean for British subjects), ${ }^{22}$ and $3 \cdot 3-5 \cdot 1 \mathrm{mmol} / 1$.

\section{ANALYSIS OF DATA}

Data were analysed with the statistical package SPSS. ${ }^{23}$ Analysis of variance (procedure, means) and $\chi$ tests (procedure, cross tabulation) were used as appropriate. Multiple regression of measured variables against fasting and two hour post glucose load blood glucose concentrations was performed, as was stepwise logistic regression for determinants of diabetes and impaired glucose tolerance.

\section{Results}

Tables II and III show the distribution of body mass index, haemoglobin, and cholesterol concentrations. About half of the men and over a third of the women were underweight by the conventional criterion of body mass index $<20 \mathrm{~kg} / \mathrm{m}^{2}$. Body mass index was less than $17.3 \mathrm{~kg} / \mathrm{m}^{2}$ and $17.6 \mathrm{~kg} / \mathrm{m}^{2}$ in $10 \%$ of men and women respectively, reflecting severe current undernutrition. Anaemia was common in the three villages in Morogoro region (values for both sexes together were $22.1 \%$ in Melela, $31 \cdot 2 \%$ in Madizini, and $28.3 \%$ in Msolwa) and in Nyambori in Mara region (21.8\%), suggesting more long term malnutrition. Mean serum cholesterol concentrations were also significantly lower in Morogoro region, reflecting the low intake of fat and animal protein in these villages. In Mdawi (Kilimanjaro region) the cholesterol concentrations were similar to those found in an Asian Muslim community in Dar es Salaam. ${ }^{2}$

Table IV shows the crude prevalences of diabetes and impaired glucose tolerance by age groups and fifths of body mass index. No consistent relation was found between rates of diabetes and body mass index ( $\chi^{2}$ analysis for trends, NS), although the prevalence of diabetes increased with age. Similarly, there was no relation between height and rate of diabetes (table V). For the lowest tenth of body mass index $\left(<17.3 \mathrm{~kg} / \mathrm{m}^{2}\right.$ 
for men and $<17.6 \mathrm{~kg} / \mathrm{m}^{2}$ for women) prevalences of diabetes and impaired glucose tolerance were $1.5 \%$ (95\% confidence interval $0.8 \%$ to $2 \cdot 8 \%$ ) and $9 \cdot 0 \%$ $(7 \cdot 1 \%$ to $11 \cdot 2 \%)$. For the highest tenth $(>23 \cdot 1 \mathrm{~kg} / \mathrm{m}$ for men, $>24.7 \mathrm{~kg} / \mathrm{m}^{2}$ for women) the respective rates were $1 \cdot 2 \%(0 \cdot 6 \%$ to $2 \cdot 2 \%)$ and $7 \cdot 2 \%(5 \cdot 6 \%$ to $9 \cdot 2 \%)$. Impaired glucose tolerance prevalence was highest in the lowest fifths of height $(p<0.05$ both sexes; $p<0.05$ men only) and body mass index. However, when those overweight by conventional standards (body mass index $\left.\geqslant 25 \mathrm{~kg} / \mathrm{m}^{2}\right)$ were considered $(\mathrm{n}=545)$ the prevalence of diabetes was $1.9 \%$ and of impaired glucose tolerance $8.9 \%$, rising to $6.0 \%$ and $20.0 \%$ respectively in the oldest age group. No correlation was seen between diabetes or impaired glucose tolerance and haemoglobin or cholesterol concentrations.

Tables VI and VII show mean blood glucose concentrations by age groups for each fifth of body mass index and height. Both fasting and two hour blood glucose concentrations were higher for the older than the younger age groups. However, no clear pattern was seen for fasting blood glucose concentrations with either body mass index or height, although two hour glucose concentrations tended to be higher in the lower than the upper fifths $(p<0.05)$.

Table VIII shows the determinants of the fasting and two hour blood glucose values. Not surprisingly, the two hour blood glucose was the main determinant of fasting blood glucose and vice versa. Age was the other major determinant of both glucose values. Height was strongly and negatively associated with two hour blood glucose but more weakly and positively with fasting glucose. Body mass index was, however, negatively correlated with both glucose values but this was the weakest of the associations. On stepwise logistic

TABLE VI-Mean blood glucose concentrations (mmolll) by body mass index, fifths, and age groups

\begin{tabular}{|c|c|c|c|c|c|c|c|c|}
\hline \multirow{2}{*}{$\begin{array}{l}\text { Body } \\
\text { mass index } \\
(\mathrm{kg} / \mathrm{m})\end{array}$} & \multicolumn{4}{|c|}{ Fasting blood glucose } & \multicolumn{4}{|c|}{2 Hour blood glucose } \\
\hline & $\begin{array}{l}15-34 \\
\text { years }\end{array}$ & $\begin{array}{l}35-54 \\
\text { years }\end{array}$ & $\begin{array}{l}\geqslant 55 \\
\text { years }\end{array}$ & $\begin{array}{c}\text { All } \\
\text { ages }\end{array}$ & $\begin{array}{l}15-34 \\
\text { years }\end{array}$ & $\begin{array}{l}35-54 \\
\text { years }\end{array}$ & $\begin{array}{l}\geqslant 55 \\
\text { years }\end{array}$ & $\begin{array}{c}\text { All } \\
\text { ages }\end{array}$ \\
\hline \multicolumn{9}{|l|}{ Men: } \\
\hline$<18.2$ & $4 \cdot 2$ & $4 \cdot 1$ & $4 \cdot 2$ & $4 \cdot 2$ & $4 \cdot 7$ & 4.5 & 5.7 & 4.9 \\
\hline$-19 \cdot 5$ & $4 \cdot 1$ & $4 \cdot 2$ & 4.4 & $4 \cdot 2$ & $4 \cdot 4$ & $4 \cdot 3$ & 5.0 & 4.5 \\
\hline$-20 \cdot 6$ & $4 \cdot 1$ & $4 \cdot 2$ & $4 \cdot 4$ & $4 \cdot 2$ & $4 \cdot 3$ & $4 \cdot 3$ & $4 \cdot 8$ & $4 \cdot 4$ \\
\hline$-21 \cdot 8$ & $4 \cdot 0$ & $4 \cdot 3$ & $4 \cdot 2$ & $4 \cdot 1$ & $4 \cdot 2$ & $4 \cdot 4$ & $4 \cdot 6$ & $4 \cdot 4$ \\
\hline$>21.9$ & $4 \cdot 0$ & $4 \cdot 1$ & $4 \cdot 4$ & $4 \cdot 1$ & $4 \cdot 1$ & $4 \cdot 2$ & $4 \cdot 8$ & $4 \cdot 3$ \\
\hline Total & $4 \cdot 1$ & $4 \cdot 2$ & $4 \cdot 3$ & $4 \cdot 2$ & $4 \cdot 4$ & $4 \cdot 3$ & $5 \cdot 0$ & $4 \cdot 5$ \\
\hline \multicolumn{9}{|l|}{ W'omen: } \\
\hline$<18.6$ & $4 \cdot 1$ & $4 \cdot 3$ & 4.5 & $4 \cdot 2$ & $4 \cdot 7$ & $5 \cdot 1$ & $5 \cdot 3$ & $5 \cdot 0$ \\
\hline$-20 \cdot 1$ & $4 \cdot 0$ & $4 \cdot 2$ & $4 \cdot 3$ & $4 \cdot 1$ & $4 \cdot 7$ & $4 \cdot 8$ & $5 \cdot 1$ & $4 \cdot 8$ \\
\hline$-21 \cdot 5$ & $4 \cdot 0$ & 4.2 & $4 \cdot 5$ & $4 \cdot 1$ & $4 \cdot 6$ & $4 \cdot 9$ & $5 \cdot 4$ & 4.8 \\
\hline$-23 \cdot 2$ & $4 \cdot 0$ & $4 \cdot 1$ & $4 \cdot 5$ & $4 \cdot 1$ & $4 \cdot 6$ & 4.7 & $5 \cdot 2$ & 4.7 \\
\hline$>23 \cdot 2$ & 3.9 & $4 \cdot 2$ & $4 \cdot 4$ & $4 \cdot 1$ & $4 \cdot 5$ & 4.9 & $5 \cdot 5$ & $4 \cdot 8$ \\
\hline Total & $4 \cdot 0$ & $4 \cdot 2$ & $4 \cdot 4$ & $4 \cdot 1$ & $4 \cdot 6$ & $4 \cdot 9$ & $5 \cdot 3$ & $4 \cdot 8$ \\
\hline
\end{tabular}

TABLE VII-Mean blood glucose concentrations (mmol/) by height, fifths, and age groups

\begin{tabular}{|c|c|c|c|c|c|c|c|c|}
\hline \multirow[b]{2}{*}{$\begin{array}{l}\text { Height } \\
\text { (m) }\end{array}$} & \multicolumn{4}{|c|}{ Fasting blood glucose } & \multicolumn{4}{|c|}{2 Hour blood glucose } \\
\hline & $\begin{array}{l}15-34 \\
\text { years }\end{array}$ & $\begin{array}{l}35-54 \\
\text { years }\end{array}$ & $\begin{array}{l}\geqslant 55 \\
\text { years }\end{array}$ & $\begin{array}{c}\text { All } \\
\text { ages }\end{array}$ & $\begin{array}{l}15-34 \\
\text { years }\end{array}$ & $\begin{array}{l}35-54 \\
\text { years }\end{array}$ & $\begin{array}{l}\geqslant 55 \\
\text { years }\end{array}$ & $\begin{array}{c}\text { All } \\
\text { ages }\end{array}$ \\
\hline \multicolumn{9}{|l|}{ Men: } \\
\hline$<1.58$ & $4 \cdot 2$ & $4 \cdot 2$ & $4 \cdot 2$ & $4 \cdot 2$ & $4 \cdot 6$ & $4 \cdot 8$ & $5 \cdot 4$ & $4 \cdot 8$ \\
\hline-1.63 & $4 \cdot 0$ & $4 \cdot 2$ & $4 \cdot 3$ & $4 \cdot 2$ & $4 \cdot 4$ & $4 \cdot 5$ & $5 \cdot 0$ & $4 \cdot 6$ \\
\hline-1.665 & 4.0 & $4 \cdot 1$ & $4 \cdot 3$ & $4 \cdot 1$ & $4 \cdot 3$ & $4 \cdot 3$ & $4 \cdot 9$ & $4 \cdot 4$ \\
\hline-1.71 & $4 \cdot 1$ & $4 \cdot 1$ & $4 \cdot 5$ & $4 \cdot 2$ & $4 \cdot 3$ & $4 \cdot 1$ & $4 \cdot 9$ & $4 \cdot 4$ \\
\hline$>1.71$ & $4 \cdot 1$ & $4 \cdot 2$ & $4 \cdot 3$ & $4 \cdot 2$ & $4 \cdot 2$ & $4 \cdot 2$ & $4 \cdot 9$ & $4 \cdot 3$ \\
\hline Total & $4 \cdot 1$ & $4 \cdot 2$ & $4 \cdot 3$ & $4 \cdot 2$ & $4 \cdot 4$ & $4 \cdot 3$ & $5 \cdot 0$ & 4.5 \\
\hline \multicolumn{9}{|l|}{ Women: } \\
\hline$<1.505$ & $4 \cdot 0$ & $4 \cdot 2$ & $4 \cdot 4$ & $4 \cdot 2$ & $4 \cdot 7$ & $5 \cdot 1$ & $5 \cdot 5$ & $5 \cdot 0$ \\
\hline-1.545 & $4 \cdot 0$ & $4 \cdot 1$ & $4 \cdot 5$ & $4 \cdot 1$ & $4 \cdot 6$ & $4 \cdot 9$ & $5 \cdot 3$ & $4 \cdot 8$ \\
\hline-1.575 & $4 \cdot 0$ & $4 \cdot 2$ & $4 \cdot 4$ & $4 \cdot 1$ & $4 \cdot 6$ & 4.9 & $5 \cdot 1$ & $4 \cdot 8$ \\
\hline-1.615 & $4 \cdot 0$ & $4 \cdot 3$ & $4 \cdot 4$ & $4 \cdot 1$ & $4 \cdot 6$ & 4.9 & $5 \cdot 1$ & $4 \cdot 8$ \\
\hline$>1.615$ & $4 \cdot 0$ & $4 \cdot 2$ & $4 \cdot 5$ & $4 \cdot 1$ & $4 \cdot 6$ & $4 \cdot 7$ & $5 \cdot 1$ & $4 \cdot 7$ \\
\hline Total & $4 \cdot 0$ & $4 \cdot 2$ & $4 \cdot 4$ & $4 \cdot 1$ & $4 \cdot 6$ & $4 \cdot 9$ & $5 \cdot 3$ & $4 \cdot 8$ \\
\hline
\end{tabular}

TABLE VII--Multiple stepatse regression analysis of blood glucose concentration, body mass index, height, age, and sex with fasting blood glucose and two hour blood glucose concentrations as dependent variablc

\begin{tabular}{|c|c|c|}
\hline Variable & T value & Significance \\
\hline \multicolumn{3}{|c|}{ Fusting blood glucose } \\
\hline 2 Hour blood glucose & 45.09 & $<0.0001$ \\
\hline Age & $8 \cdot 66$ & $<0.0001$ \\
\hline Sex & -3.03 & 0.0024 \\
\hline Height & $3 \cdot 70$ & $0 \cdot 0002$ \\
\hline Body mass index & -2.52 & 0.0016 \\
\hline Constant & $14 \cdot 28$ & \\
\hline \multicolumn{3}{|c|}{ Multiple $\mathrm{R}=0 \cdot 478, \mathrm{R}=0.228$} \\
\hline \multicolumn{3}{|c|}{2 Hour blood gluorse } \\
\hline Fasting blood glucose & 45.09 & $<0.0001$ \\
\hline Height & $-8 \cdot 69$ & $<0.0001$ \\
\hline Age & $8 \cdot 19$ & $<0.0001$ \\
\hline Sex & $5 \cdot 73$ & $<0.0001$ \\
\hline Body mass index & -2.57 & 0.0101 \\
\hline Constant & $9 \cdot 27$ & \\
\hline
\end{tabular}

Multiple $\mathrm{R}=0 \cdot 466, \mathrm{R}:=0 \cdot 217$

regression of determinants of diabetes and impaired glucose tolerance, including height, body mass index, age, and sex, only age was significantly associated with diabetes and impaired glucose tolerance.

\section{Discussion}

This study indicates that in Tanzania, at least, diabetes is not more common in the most undernourished members of the population, and that it is much less common than in well nourished Western populations. ${ }^{25}$ Indeed, the Tanzanian rates of diabetes and impaired glucose tolerance may be overestimates as they were based on a single oral glucose tolerance test in a population unaccustomed to venepuncture..$^{26}$

Rates of diabetes in the two lowest fifths of body mass index (both below $20 \mathrm{~kg} / \mathrm{m}^{2}$ ) and the lowest tenth were not significantly different from rates in the upper two fifths $(1.3 \%$ and $0.8 \%, 1.0 \%$ and $0.9 \%$ respectively). There was a trend for those of lowest height, reflecting long term poor nutrition, to have higher rates of diabetes and impaired glucose tolerance. It should be emphasised that these trends reflected a tiny number of cases-2-3 per 1000 population-and the effect, even if statistically significant, is clinically trivial. Fasting blood glucose concentrations were not significantly different among fifths of body mass index and height, although two hour blood glucose values and impaired glucose tolerance rates, which are defined by two hour glucose values, were higher in the lowest fifths than in the higher fifths for both body mass index and height. This difference could either be a genuine effect or, more likely in the absence of any change in fasting blood glucose, reflect the fact that all subjects, irrespective of weight, were given $75 \mathrm{~g}$ glucose-that is, the lightest individuals received the highest glucose load per kilogram weight. ${ }^{27}$ Short term malnutrition could also have contributed..$^{28}$ This also fits the observation that although there was a negative correlation between height and two hour blood glucose, a weak positive relation was seen for fasting glucose (table VIII).

\section{IMPORTANCE OF UNDERNUTRITION}

Evidence that undernutrition itself may be diabetogenic comes mainly from studies in animals, ${ }^{28}{ }^{29}$ adults, ${ }^{3131}$ and children ${ }^{323}$ with protein energy malnutrition in whom functional abnormalities in insulin secretion and glucose tolerance have been shown. Recently, animal studies have suggested that severe maternal protein deficiency in pregnancy results in B cell damage and subsequent diabetes in the offspring $^{3+}$; others have suggested that low birth weight in humans predicts the development of impaired 
glucose tolerance and non-insulin dependent diabetes mellitus in later life. ${ }^{35}$ We have no evidence to support or refute these suggestions, although the overall low prevalence of non-insulin dependent diabetes and impaired glucose tolerance makes any important contribution unlikely. Overnutrition in later life, which increases insulin requirement, may uncover a defect in the secretory capacity of B cells, which could explain the current epidemic of non-insulin dependent diabetes mellitus in developing countries that have recently become more affluent. ${ }^{36}$ This is a separate problem from that of malnutrition related diabetes mellitus, in which current as well as previous malnutrition is said to be a key component.

Postmortem studies in humans have also shown that severe kwashiorkor may be associated with structural changes in the pancreas. ${ }^{37}$ It is uncertain, however, whether the functional and structural changes are sufficiently severe to lead to permanent diabetes. Our results suggest that they are not.

Undernutrition has also been suggested as a predisposing factor in tropical chronic calcific pancreatitis, possibly associated with recurrent attacks of gastroenteritis. ${ }^{35}$ In Africa this disease has been described mainly in young adults in Uganda ${ }^{38}{ }^{34}$ and Nigeria. ${ }^{10}$ If undernutrition were a key factor in the development of tropical chronic calcific pancreatitis it would be expected to be commoner than it is in other parts of Africa where malnutrition is more widespread. ${ }^{+1}$ Alcohol is almost certainly an important cause of chronic pancreatitis, ${ }^{+2}$ but does not account for calcific pancreatitis in the young.

\section{ASSESSMENT OF NUTRITION}

The populations we have studied could be said to be insufficiently malnourished or undernourished for an insufficient time to induce irreversible $B$ cell failure. It is also possible that those who developed diabetes died undiagnosed. This possibility, while unlikely, cannot definitely be excluded. The assessment of undernutrition in adults is difficult. No single satisfactory measure exists, but body mass index is possibly the best available. About $40 \%$ of our population were underweight according to accepted body mass index standards. Some authorities in Africa have proposed that the lower limit of normal for body mass index in African subjects be reduced ( $T$ Johnson, personal communication). But there is no evidence on which to base this proposal, and studies have yet to be conducted which relate body mass index to mortality in Africans. We therefore believe that the low values for body mass index found in our study reflect undernourishment in a large proportion of the population. The low haemoglobin concentrations in four of the eight villages also point to poor nutritional state.

Assessment of chronic protein energy malnutrition is even more difficult. Many experts regard the ultimate height of populations as the most satisfactory index of chronic undernutrition during the period of growth and development. Genetic factors play a large part in the determination of height, but it is now widely accepted that improvement in the nutritional state of a nation's population increases the mean height of its citizens. ${ }^{+3+t}$ The mean height of the study population was significantly less than the mean height of a British population. ${ }^{+5}$ In addition, evidence of poor nutrition in children in several areas in which the studies were performed $^{1617}$ also provided circumstantial evidence that the adults had grown up in an adverse nutritional environment. This is therefore strong evidence that both current and past undernutrition were common in the study populations. Importantly, there were also no differences between prevalence of diabetes and impaired glucose tolerance in the least advantaged and the most advantaged villages.
The proponents of malnutrition related diabetes have also suggested that while undernutrition may not lead to the development of diabetes it may increase the vulnerability of the pancreas to harmful environmental factors. ${ }^{4}$ Cyanide from the consumption of inadequately processed bitter cassava has been proposed as a possible environmental toxin. ${ }^{40}$ We found, however, that rates of diabetes in one village where the inhabitants were exposed to high levels of dietary cyanogenic glucosides from consumption of insufficiently processed cassava were not significantly different from rates in the seven other villages. ${ }^{47}$

Our studies of glucose tolerance in rural Tanzania have therefore failed to confirm the widely held hypothesis that undernutrition by itself or undernutrition and environmental toxins are a significant cause of diabetes in undernourished populations.

We thank the Ministry of Health, United Republic of Tanzania, for permission to carry out the study; the communities for their cooperation and participation; and the director general of Muhimbili Medical Centre, the British Council, and the British Diabetic Association for support. Thanks are also due to the staff of the department of biochemistry, Muhimbili Medical Centre, and department of medicine, University of Newcastle upon Tyne, for technical help.

1 WHO Study Group. Diabetes mellitus. WHO Technical Report Scries 1985 727.

2 Bajaj JS. Diabetes mellitus in developing countries. New Delhi: Interprint, 1984.

3 McMillan DE, Geevarghese PJ. Dietary cyanide and tropical malnutrition diabetes. Diabetes Care 1979;2:202-8.

4 Rao RH. Diabetes in the undernourished: coincidence or consequence? Endocr Rev 1988:9:67-87. 5 Keen H, Ekoe JM. The geography of diabetes mellitus. Br Med Bull

6 Abu-Bakare A, Gill GV, Taylor R, Alberti KGMM. Tropical or malnutrition related diabetes: a real syndrome? Lancet 1986;i:1 135-8.

Khardori R. Undernutrition and diabetes. Diabetes Care 1985;8:302

West KM. Epidemiology of diabetes and its vascular complications. New York: Elsevier, 1978:231-73.

9 Himsworth HP. Diet in the aetiology of human diabetes. Proc $R$ Soc Med 1949;42:323-6.

10 West KM, Kalbfleisch JM. Influence of nutritional factors on prevalence of diabetes. Diabetes 1971;20:99-108.

11 Gupta OP, Dave SK, Gupta PS, Hegde HS, Agarwal SB, Joshi MN, et al. Aetiological factors in the prevalence of diabetes in urban and rural populations in India. In: Baba S, Goto Y, Fukui I, eds. Diabetes mellitus in Asia. Kyoto, Excerpta Medica, 1976:23-4. (Excerpta Medica International Asia. Kyoto, Excerpta
Congress Series 390.)

12 Rao RH. The role of undernutrition in the pathogenesis of diabetes mellitus. Diabetes Care 1984;7:595-601.

13 McLarty DG, Kitange HM, Mtangi BL, Makene WJ, Swai ABM, Masuki G, et al. Prevalence of diabetes and impaired glucose tolerance in rural Tanzania. Lancet 1989;i:871-5.

14 Kimati VP, Scrimshaw NS, The nutritional status of Tanzanian children: a cross-sectional anthropometric community survey report. East Afr Med $\mathcal{f}$ 1985;62:105-17

15 Mrisho F. Epidemiology of malnutrition in Tanzania. In: Italian Medical Team, ed. Maluatrition in Tanzania. Padua: CUAMM, International College for Health Cooperation in Developing Countries, 1986:7-16.

16 Tanner M, Burnier E, Mayombana C, Betschart B, de Savigny D, Marti HP, et al. Longitudinal study on the health status of children in a rural Tanzanian community: parasitoses and nutrition following control measures against intestinal parasites. Acta Trop 1987;44:137-74.

17 UNICEF. Preliminary report. Hai nutrition campaign. Dar es Salaam: UNICEF, 1987

18 Allain CC, Poon LS, Chan CSG, Richmond W, Fu PC. Enzymatic determination of total serum cholesterol. Clin Chem 1974;20:470-5.

19 Fleming AF. The blood. In: Parry EHO, ed. Principles of medicine in Africa Oxford: Oxford University Press, 1984:705-60.

20 Study Group of the European Atherosclerosis Society. Strategies for the prevention of coronary heart disease: a policy statement of the European Atherosclerosis Society. Eur Heart $\mathcal{F} 1987 ; 8: 77-8$.

21 National Heart, Lung, and Blood Institute Workshop. Recommendations regarding public screening for measuring blood cholesterol. Arch Intern Med 1989;149:2650-4.

22 Mann JI, Lewis B, Shepherd J, Winder AF, Fenster S, Rose I, ct al. Blood lipid concentrations and other cardiovascular risk factors: distribution, prevalence, and detection in Britain. BMF 1988;296:1702-6.

23 SPSS, Norusis MJ. SPSS/PC: SPSS for the IBM PC/XT/AT. Chicago: SPSS, 1986.

24 Alberti KGMM, Swai ABM, McLarty DG, Kitange HM, Chuwa LM, Sherrif $\mathrm{F}$, et al. Plasma lipids and glucose intolerance in different communities and racial groups in Tanzania. Diab Res Clin Pract 1988;5(suppl 1): S449.

25 Harris M, Hadden WC, Knowler WC, Bennett PH. Prevalence of diabetes and impaired glucose tolerance and plasma glucose levels in US population aged
intris 20-74 years. Diabetes 1987;36:523-34

26 Swai ABM, McLarty DG, Kitange HM, Kilima PM, Masuki G, Mtangi BL, et al. Impaired glucose tolerance. A methodological myth? A study in Tanzania. Diabetes 1991;40:516-20.

27 West KM. Epidemiology of diabetes and its vascular complications. New York: Elsevier, 1978:74-80

28 Heard CRC, Turner MR. Glucose tolerance and related factors in dogs fed diets of suboptimal protein value. Diabetes 1967;16:96-107. 
29 Swenne I, Crace CJ, Milner RDG. Persistent impairment of insulin secretory response to glucose in adult rats after limited period of protein-calor malnutrition early in life. Diabetes $1987 ; 36: 454-8$

30 Gopalan C, Venkatachalam PS, Rao KS, Menon PS. Studies on nutritional edema: clinical, biochemical and pathological observations. Indian $7 \mathrm{Med}$ Sci 1952;6:277-95.

31 Smith SR, Edgar PJ, Pozefsky T, Chetri MK, Prout TE. Insulin secretion and glucose tolerance in adults with protein-calorie malnutrition. Metabolism 1975;24:1073-84

32 Cook GC. Glucose tolerance after kwashiorkor. Nature 1967;215:1295-6.

33 Becker DJ, Pimstone BL Hansen JDL Hendricks S. Insulin secretion in protein-calorie malnutrition. 1. Quantitative abnormalities and response to Preatment. Diabutes 1971;20:542-51.

34 Snoeck A, Remacle C, Reusens B, Hoet JJ. Effect of a low protein die: during pregnancy on the fetal rat endocrine pancreas. Biol Neonate 1990;57:107-18.

35 Hales CN, Barker DJP, Clark PMS, Cox LJ, Fall C, Osmond C, et al. Fetal and infant growth and impaired glucose tolerance at age 64. BMF 1991;303:1019-22

36 Bennett PH, Bogardus C, Tuomilehto J, Zimmet P. Epidemiology and natural history of NIDDM: non-obese and obese. In: Alberti KGMM, DeFronzo $\mathrm{RA}$, Keen $\mathrm{H}$, Zimmet $\mathrm{P}$, eds. International textbook of diabetes mellitus. Chichester: John Wiley and Sons, 1992:147-76.

37 Davies JNP. The essential pathology of kwashiorkor. Lancet 1948;i:317-20.

38 Nwokolo C, Oli J. Pathogenesis of juvenile tropical pancreatitis syndrome. Lance 1980;i:457-9.
39 Shaper AG. Chronic pancreatic disease and protein malnutrition. Lance 1960;i:1223-4.

40 Olurin EO, Olurin O. Pancreatic calcification: a report of 45 cases. BMJ 1969;iv:534-9

41 Lester FT. A search for malnutrition diabetes in an Ethiopian diabetic clinic. International Diabetes Foundation Bulletin 1984;29:14-6.

42 McLarty D, Pollitt C, Swai ABM. Diabetes in Africa. Diabetic Med 1990;? 670-84

43 Tanner JM, Healy MJR, Lockhart RD, MacKenzie JD. Whitehouse RH. Aberdeen growth study. 1. The prediction of adult body measurements from measurements taken each year from birth to 5 years. Arch Dis Child 1956;31:372-81.

44 Whincup PH, Cook DG, Shaper AG. Social class and height. BMF 1988;197

45 Walker M, Shaper AG, Wannamethee G. Height and social class in middleaged British men. F Epidemiol Commumity Health 1988;42:299-303.

46 Pitchumoni CS, Jain NK, Lowenfels AB, DiMagno EP. Chronic cyanide poisoning: unifving concept for alcoholic and tropical pancreatitis. Pancreas 1988;3:220-2

47 Swai ABM, McLarty DG, Mtinangi BKL, Tatala S, Kitange HM, Mlingi N, et al. Diabetes is not caused by cassava toxicity: a study in a. Tanzania community. Diabetes Care (in press).

(Accepted 8 Siptember 1992)

\title{
Decision to treat mild hypertension after assessment by ambulatory monitoring and World Health Organisation recommendations
}

\author{
Gilles Chatellier, Christiane Battaglia, Jean-Yves Pagny, Pierre-François Plouin, Joël Ménard
}

\begin{abstract}
Abstrtact
Objective-To determine if one ambulatory blood pressure recording over 12 hours could detect those patients with mild hypertension who needed treatment according to the World Health OrganisationInternational Society of Hypertension (ISH) guidelines based on the casual measurement of diastolic blood pressure at successive visits to a clinic.

Design-Comparison of decision to treat based on one ambulatory measurement over 12 hours and standard blood pressure measurements over six months in the same patients.

Setting-Outpatient hypertension clinic.

Subjects-130 men and women with diastolic blood pressure of 90-104 $\mathrm{mm} \mathrm{Hg}$ at second visit to clinic.

Main outcome measures-Blood pressure measurements over six months. Measurement from ambulatory monitoring. Decision to treat.

Results-Of the 130 patients included, 108 were followed up over the six months. Treatment was started according to WHO-ISH criteria in 44 (13 at the third visit, 13 at the fourth, 18 at the fifth). According to the selected criteria for ambulatory blood pressure monitoring 41 patients would have been treated. Both methods agreed that the same 27 patients required treatment and the same 50 did not, but they did not agree in 31 patients. When calculated at the optimal diastolic blood pressure threshold determined by a receiver operating characteristic curve, the sensitivity, specificity, and positive predictive value of ambulatory blood pressure monitoring were $71 \% \quad(95 \%$ confidence interval $57 \%$ to $84 \%), 82 \%(72 \%$ to $92 \%)$, and $66 \%$ $(51 \%$ to $81 \%)$, respectively.
\end{abstract}

Conclusion-If the WHO-ISH criteria are accepted as the standard for deciding to treat patients with mild hypertension the predictive value of one ambulatory blood pressure recording over 12 hours is too low to detect with confidence those patients who need treatment when managed according to these criteria.

\section{Introduction}

In patients with mild hypertension the decision to treat is an important issue that has again come to the fore with the availability of several new techniques for measuring blood pressure. The rise in blood pressure induced by the act of measurement itself, referred to as the "white coat reaction," may lead to a false diagnosis of hypertension.' This is one reason why $40 \%$ of the patients included in large scale hypertension trials subsequently became normotensive with placebo. ${ }^{23}$

The guidelines from the World Health OrganisationInternational Society of Hypertension (ISH) take this into account and recommend multiple mesurements over six months before treatment is started. ${ }^{+}$The basis for such a policy is, firstly, that multiple measurements may be expected to give a closer estimation of the true blood pressure and, secondly, that repetition of measures over time may decrease the reaction induced by the visit to the doctor. This protocol is presently the gold standard for the decision to treat mild hypertension because epidemiological data, classification of hypertension, and evaluation of treatment efficacy are all based on clinic readings and because the white coat reaction has been shown to diminish considerably after four months. ${ }^{2}$ This protocol, however, is tedious, risks losing patients to follow up, and the extent of its correct use by general practitioners is unknown. Consequently, it may prove to be less useful than expected when applied in reality.

Ambulatory blood pressure monitoring is a widely accepted method of measuring blood pressure, which avoids the white coat reaction' and becomes increasingly acceptable to patients as the equipment gets smaller. The value of a single ambulatory record in predicting the need for treatment has never been compared with that of a reference method. We assessed the predictive value of one 12 hour ambulatory blood pressure record for the decision to treat patients with mild hypertension by comparing it with the decision made during the six months' follow up in the clinic, according to the WHO-ISH protocol. ${ }^{+}$

\section{Patients and methods}

A total of 154 men and women who were referred to the hypertension clinic of the Broussais University Hospital for evaluation of their hypertension were screened for inclusion in the present study. The inclusion criteria were (a) casual diastolic blood 